\title{
A Cross-Layer Routing Protocol Based on Location Information for Multi-hop Wireless Ad hoc Networks with Directional Antenna
}

\author{
Tai-bin HUANG ${ }^{*}$ and Lei LEI
}

College of Electronic and Information Engineering, Nanjing University of Aeronautics and Astronautics, Nanjing[2111000], China;

Keywords: Multi-hop wireless ad hoc networks, Directional antennas, Cross-layer design.

\begin{abstract}
In multi-hop wireless ad hoc networks, communications with directional antennas can effectively improve the transmission range of the node. Most of existing directional routing protocols adopt the four-way handshaking mechanism which transmits and receives with directional and omnidirectional antennas respectively. As a result, the transmission range of the node is tremendously limited due to the underutilization of the directional antenna gain of the receiver. To solve this problem, in this paper, a directional cross-layer routing protocol based on location information for multi-hop wireless ad hoc networks is proposed. In this protocol, we propose a novel handshaking mechanism to achieve directional to directional beam alignment. Further, the source node chooses the optimal directional to directional Routing by a cross-layer manner. Simulation results show that the protocol can achieve the better throughput and end-to-end delay performance compares with that obtained by the existing directional routing protocol.
\end{abstract}

\section{Introduction}

In the multi-hop wireless ad hoc networks[1-2], directional transmission can not only increase the transmission range, but also reduce the number of route hops, the end-to-end delay and improve the utilization of the network space. However, communication with directional antenna causes the new problems of directional access, such as "directional hidden terminal"[3-4] and "deafness"[5-6] etc. These problems will affect routing discovery and maintenance. For the network layer, although communication with directional antenna can reduce the flooding area of route request (RREQ) packets, it increases the failure probability of route discovery, and leads to increased routing overhead and redundant routing reconfiguration. All of the above problems will cause network performance degradation.

\section{Related Work}

Aiming at the problems of "directional hidden terminal" caused by the difference of directional gain and omnidirectional gain in antenna operation and "deafness" of the node, the author proposed a hybrid routing protocol based on mixed multi-path in [7], it provided that the source and forwarding nodes can find routing through omnidirectional broadcasts to achieve the link connection. By selecting the optimal route with no overlapping beam, it can reduce the interference of "directional hidden terminal" and "deafness". However, this method of route discovery will increase the average end-to-end delay of the packets. Aiming at the problem of directed flooding overhead, H. Gharavi and B. Hu proposed the Directional DSR protocol in [8]. The protocol selects the routes based on the shortest route hops, the number of overlapping 
beams and the number of repeat nodes, which can effectively reduce the interference between paths. However, this protocol uses the way of scanning between beams to find routes will increase the cost of route discovery. In [9], the author proposed a directional routing protocol (DRP). In the process of route discovery, it is preferred to find the route between the previous antenna beam and the adjacent beams. After the link is interrupted, two-hop route recovery is used to rebuild the connection. The advantage of this protocol is improving the probabilities of route discovery, reducing additional cost of route reconfiguration. However, the handshake mechanism leads to limited transmission range.

Therefore, we propose a directional cross-layer routing protocol based on location information for multi-hop wireless ad hoc network (DCLRP-L). In this protocol, after the node finds the route, it will select the shortest and maximum space division route by clipping. The single-hop transmission range can be maximized by adopting the handshake mechanism of relay RTS. Simulation results show that, DCLRP-L has good performance of throughput and end-to-end delay compared with DRP.

\section{DCLRP-L Protocol Description}

\section{Design of MAC Layer}

In the existing directional MAC protocols, D-O mode is widely used to exchange control frames to achieve beam alignment resulting in limited communication range of single-hop. To overcome this limitation, we propose a new beam alignment strategy to maximize the communication range of single-hop.

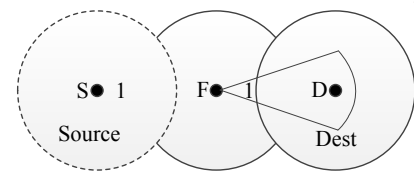

a) DO-RTS Interaction

\begin{tabular}{|c|c|c|c|}
\hline DEST & BEAM & F-RTS-ID & DEAF-TIME \\
\hline F & 1 & F & 0 \\
\hline D & 1 & F & 0 \\
\hline & & & \\
\hline
\end{tabular}

b) DNT of Node S

Figure 1. DO-RTS Interaction and Directional Neighbor Table

We assume that nodes S, F, D are separated by $500 \mathrm{~m}$ and node S adopts D-O mode to finds the route as $\{F, D\}$ in Figure 1(a).Than we clip the route to $\{\mathrm{D}\}$, and store node $\mathrm{F}$ as forwarding node (F-RTS-ID) of DO-RTS in the directional neighbor table (DNT) in Figure 1(b). In addition, every node also maintains the beam index (BEAM) and deaf time (DEAF-TIME) of other nodes in DNT.

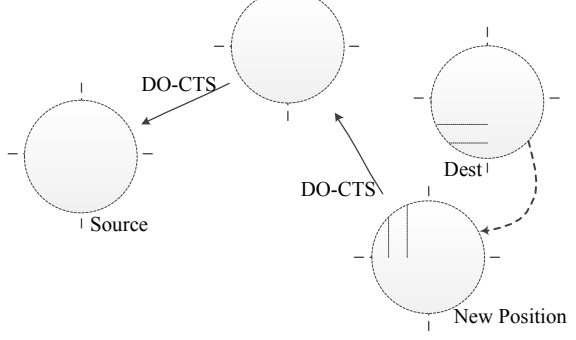

Figure 2. Relaying DO-CTS
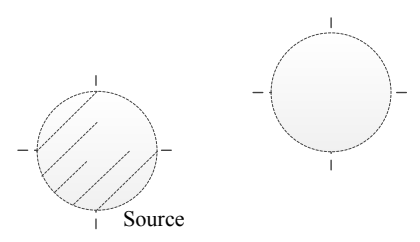

Figure 3. Transmitting Tip-RTS and Tip-CTS

As shown in Figure 2, when node $\mathrm{S}$ has data to send, it sends DO-RTS (frame structure in Figure 4(a)) through beam 4 firstly, and the parameter of Wait CTS Beam is set to 4, Forward DA is F, Forward SA is S, MAC status is set to wait for DD-CTS (frame structure is same as CTS in 802.11). If node F is a relay node, it relays the DO-RTS according to the destination information stored in the DNT. Otherwise, it updates the directional NAV (DNAV) and discards the DO-RTS. Node D receives the DO-RTS from node F and determines that the DD-CTS should be replied in beam 2 
based on the location information of the source node in the DO-RTS, and the beam 2 of node D and beam 4 of the source node saved in Wait CTS Beam can achieve beam alignment. DD-CTS, DD-DATA and DD-ACK are transmitted with D-D mode. Now, the single-hop transmission range of data is $1000 \mathrm{~m}$. If the beam cannot be aligned (Node D moves to the new position), node $\mathrm{D}$ calculates that antenna of node $\mathrm{S}$ should switch to beam 1 for alignment, and assigns beam 1 to the parameter of Switch Bean To in DO-CTS (frame structure is shown in Figure 4 (b)). Then, the DO-CTS is relayed through node $\mathrm{F}$ and the antenna is maintained in direction 3 for data. Node $\mathrm{S}$ waits for DD-CTS timeout and switch antenna operating mode to omnidirectional, MAC state is set to wait for DO-CTS. When node S receives DO-CTS, it switches antenna to the direction assigned in the parameter of Switch Bean To and sends DD-DATA. This can reduce the number of route reconfiguration caused by node's movement and realize beam alignment efficiently.

\begin{tabular}{|c|c|c|c|c|c|c|}
\hline 2 & \multicolumn{2}{|c|}{4} & 4 & 4 & 4 & 34 \\
\hline $\begin{array}{l}\text { Frame } \\
\text { Type }\end{array}$ & Dura & tion & DA & SA & FCS & Extra \\
\hline \multicolumn{7}{|c|}{ 802.11 RTS Frame } \\
\hline 4 & & 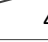 & 4 & & 4 & 24 \\
\hline & $\begin{array}{l}\text { tCTS } \\
\text { am }\end{array}$ & $\begin{aligned} \text { For } \\
D\end{aligned}$ & $\begin{array}{l}\text { ward } \\
\text { A }\end{array}$ & & $\begin{array}{l}\text { ward } \\
\text { iA }\end{array}$ & $\begin{array}{l}\text { Node } \\
\text { Location }\end{array}$ \\
\hline
\end{tabular}

a)DO-RTS

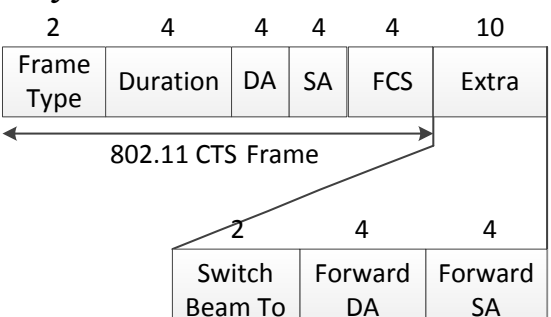

b)DO-CTS

Figure 4. Frame Structure of DO-RTS and DO-CTS

In order to avoid unnecessary back-off and collision caused by the problem of "directional hidden terminal" and "deafness", communicating nodes also broadcast the Tip-RTS and Tip-CTS frames to inform the neighboring nodes of their transmission time. As shown in Figure 3, the source and destination nodes send the Tip-RTS frame and the Tip-CTS frame in the antenna direction (the state of the direction is idle) marked by the shaded area after beam alignment. It will reduce the impact of "directional hidden terminal" and "deafness".

\section{Design of Routing Layer}

DCLRP-L is an on-demand routing protocol. In addition to DNT, it is necessary to establish and maintain a Directional Routing Table (DRT), which records D-D routing information to different destinations. The protocol is divided into three phases: 1) route discovery phase, 2 ) route processing phase, 3 ) route maintenance phase.

Route Discovery Phase. As shown in Figure 5, if node A has no information of node F in DNT, directional scanning is performed, otherwise, the source node finds the route in the direction of the destination recorded in the DNT and the adjacent direction firstly. After receiving the RREQ, the relay node continues scanning and forwarding (except for the direction of receiving RREQ and busy channel). After receiving the RREQ, node F replies RREP according to the reverse path in RREQ and adds the location information of node $F$ and relay nodes are also required. Now, the route in RREP is: $\{\mathrm{B}, \mathrm{C}, \mathrm{D}, \mathrm{E}, \mathrm{F}\}$.

Route Processing Phase. The routing information carried in RREP is a D-O route. In Figure 5, node A cuts the D-O route $\{\mathrm{B}, \mathrm{C}, \mathrm{D}, \mathrm{E}, \mathrm{F}\}$ into the $\mathrm{D}-\mathrm{D}$ route $\{\mathrm{D}, \mathrm{F}\}$ according to the node's location information. The routing clipping algorithm is based on: 1) the shortest route, 2) the highest space division. The process is as follows:

Step 1: Calculating the distance between A and F according to the location information in RREP and divide the maximum range 1110 by D-D mode to estimate the minimum hop (Min-Hop) of D-D routes. 
Step 2: According to the location information of the relay nodes, node A selects all possible relay nodes to make up multiple available D-D routes. If the number of available D-D route is 0, Min-Hop will be incremented by 1 to continue with step 2 .

Step 3: Calculating the space division count (SDC) for all possible D-D routes and selecting the best D-D route with maximum SDC.

SDC is used to calculate the number that consecutive two-hop nodes will not interfere with each other when communicating on this link from the source to the destination. SDC is used to calculate the number that consecutive two-hop nodes will not interfere with each other when communicating on this link from the source to the destination. The high SDC route will cause litter interference on this link.

Through the first two steps, we can get three effective D-D routes: route $1\{\mathrm{C}, \mathrm{E}, \mathrm{G}\}$, route $2\{\mathrm{~B}, \mathrm{E}, \mathrm{G}\}$ and route $3\{\mathrm{D}, \mathrm{E}, \mathrm{G}\}$ in Figure 6 . For route 1, node A sends data to node $\mathrm{C}$ through beam 1 , and then node $\mathrm{C}$ forward the data to node $\mathrm{E}$. When the communication is established between node $\mathrm{C}$ and $\mathrm{E}$, node $\mathrm{A}$ has new data to send and has not received Tip-RTS of node $\mathrm{C}$ in the same time. Node A sends DO-RTS to node $\mathrm{C}$ in the beam 1 to try new communication, at this time, beam 1 of node $\mathrm{A}$ and beam 7 of node $\mathrm{C}$ is aligned, the DO-RTS of node $\mathrm{A}$ will interfere with the communication between node $\mathrm{C}$ and $\mathrm{E}$. So the SDC of $\{\mathrm{A}, \mathrm{C}, \mathrm{E}\}$ is zero. For next two-hop nodes $\{\mathrm{C}$, $\mathrm{E}, \mathrm{G}$ \}, since the beam 1 of node $\mathrm{C}$ and the beam 6 of node $\mathrm{G}$ are misaligned, the SDC is 1 and the total SDC is the sum of all the adjacent two-hop nodes on this link. Therefore, the SDC of route 1 is 1 . Similarly, the total SDC of route 2 is 2 and the total SDC of route 3 is 1 . Therefore, in order to avoid the interference between neighboring two-hop nodes, we select route 2 as the best D-D route.

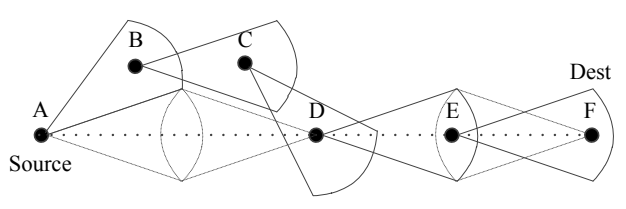

Figure 5. Route Discovery Process Figure

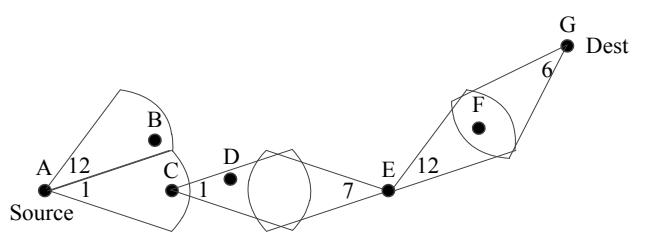

Figure 6. Calculation of Best D-D Route

Route Maintenance Phase. In the dynamic topology, the route may be broken because of the movement of nodes. DCLRP-L adopts the method of local route recovery to restore the link. When the link is interrupted at one relay node, it will find the path to the second hop node locally after its own and inform the source node of the new route though the route error packet (RERR).

\section{Performance Evaluation}

In this section, DCLRP-L and DRP protocol are analyzed and compared in network simulation environment of GloMoSim[10]. We emphatically focus on routing hops, average end-to-end delay and throughput. The protocol of transport layer is UDP, the beam width of antenna is $60^{\circ}$ and other simulation parameters are shown in Table 1.

Table 1. Simulation Parameters

\begin{tabular}{|l|l|l|l|}
\hline Parameter & Value & Parameter & Value \\
\hline Bandwidth & $1 \mathrm{Mbps}$ & D-D Range & $1110 \mathrm{~m}$ \\
\hline Antenna Mode & Switched beam & SINR & $10 \mathrm{~dB}$ \\
\hline O-O Range & $300 \mathrm{~m}$ & Packet Size & 1024 bytes \\
\hline D-O Range & $570 \mathrm{~m}$ & Application & CBR \\
\hline
\end{tabular}




\section{Static Topology}

There are 49 nodes in the network distributed in a square area with a length of 3000 meters. The distance between each two horizontal or vertical adjacent nodes is $500 \mathrm{~m}$. A CBR flow is generated in the application layer. We choose different seed values for simulation and record average end-to-end delay and throughput of DCLRP-L and DRP. The simulation results are shown in Figure 7.

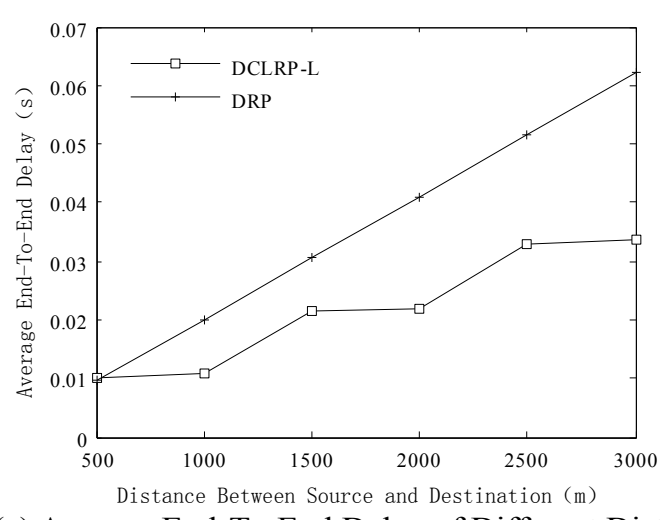

(a) Average End-To-End Delay of Different Distance

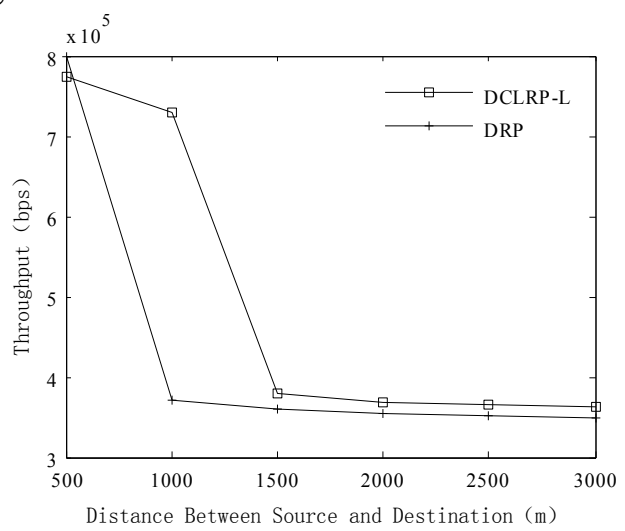

(b) Throughput of Different Distance

Figure 7. Route Hop, Average End-To-End Delay and Throughput in Static Topology

Compared with DRP, DCLRP-L can intelligently cut D-O route to D-D route and adopt the handshake mechanism of relaying DO-RTS to achieve beam alignment for two distant nodes. It can be seen that DCLRP-L can reduce average end-to-end delay compared to DRP by analyzing the graph in Figure 7 (a). As the distance increases between the source and the destination, the throughput of DCLRP-L decreases slowly compared with DRP through Figure 7 (b).

\section{Dynamic Topology}

There are 64 nodes are randomly distributed in a square area with a length of 2000 meters in the network. Two CBR flows are generated in the application layer. We can get some data such as route hops and throughput in the case of different seed values.

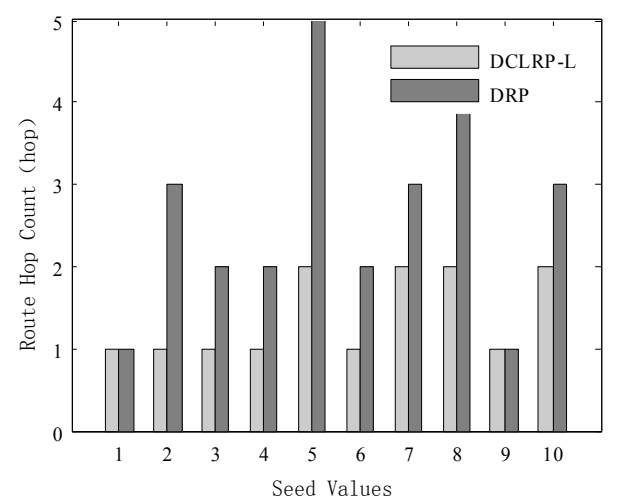

a) Route Hop with Different Seed Values

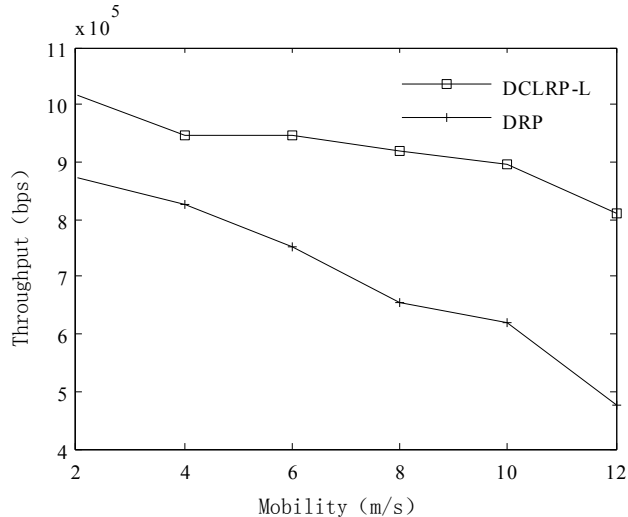

b) Throughput with Different Mobility of Nodes

Figure 8. Hop Length and Throughput in Dynamic Topology

Since the speed of node's mobility increases, the probability of routing failures is also increases. DCLRP-L adds the route processing stage to ensure that the protocol achieves the shortest route hops, better throughput and the throughput of DCLRP-L drops slowly with respect to DRP in Figure 8 (a) and (b). 


\section{Conclusions}

In summary, DCLRP-L which is proposed above has good performance. The design of the routing protocol is combined with the MAC layer design. It can obtain the shortest $\mathrm{D}-\mathrm{D}$ route with the highest SDC by cutting the $\mathrm{D}-\mathrm{O}$ route and the advantages that the communication range with directional antenna is distant is fully exploited with the handshake mechanism of relaying DO-RTS. At the same time, the MAC layer design of DCLRP-L can reduce the number of route reconfiguration caused by node's movement and the influence of "deafness" and "directional hidden termination". Simulation results show that DCLRP-L reduces the average end-to-end delay and improves the throughput performance compared with DRP. In a dynamic topology, DCLRP-L also has advantages in terms of throughput.

\section{Reference}

[1] Xu S, Saadawi T. Does the IEEE 802.11 MAC protocol work well in multihop wireless ad hoc networks?[J]. IEEE communications Magazine, 2001, 39(6): 130-137.

[2] Toh C K. Maximum battery life routing to support ubiquitous mobile computing in wireless ad hoc networks[J]. IEEE communications Magazine, 2001, 39(6): 138-147.

[3] Abdullah A A, Cai L, Gebali F. DSDMAC: dual sensing directional MAC protocol for ad hoc networks with directional antennas[J]. IEEE Transactions on Vehicular Technology, 2012, 61(3): 1266-1275.

[4] R R Choudhury, et al. On designing MAC protocols for wireless networks using directional antennas. IEEE Transactions on Mobile Computing, 2006, 5(5): 477-491.

[5] Subramanian A P, Das S R. Addressing deafness and hidden terminal problem in directional antenna based wireless multi-hop networks[J]. Wireless networks, 2010, 16(6): 1557-1567.

[6] Takata M, Bandai M, Watanabe T. A receiver-initiated directional MAC protocol for handling deafness in ad hoc networks[C]. IEEE International Conference on Communications, 2006, 9: 4089-4095.

[7] R Sharma, G R Kadambi. Multipath Routing Protocol to Support Dual Polarised Directional Communication for Performance Enhancement of MANETs. Communication Systems and Network Technologies (CSNT), 2015 Fifth International Conference on Gwalior, 2015:258-262.

[8] H. Gharavi and B. Hu.Directional Antenna for Multipath Ad Hoc Routing. 2009 6th IEEE Consumer Communications and Networking Conference, Las Vegas, NV, 2009:1-5.

[9] H. Gossain, DRP: An Efficient Directional Routing Protocol for Mobile Ad Hoc Networks, Parallel and Distributed Systems, IEEE Transactions on, vol. 17, 2006:1438-1541.

[10]UCLA. Glomosim: A scalable simulation environment for Wireless and Wired Network systems. HET-NETs, 2005. 